\title{
Neutropenic Enterocolitis and Rapid Spontaneous Resolution of Portal Venous Gas: A Non- Respiratory Manifestation of COVID-19
}

\author{
Mahin Rehman ${ }^{1}$, Amlish Gondal ${ }^{1}$, Salman Khan ${ }^{2}$, Najeeb U. Rehman ${ }^{3}$, Jaime Molina ${ }^{4}$ \\ 1. Internal Medicine, Guthrie Robert Packer Hospital, Sayre, USA 2. Pulmonary and Critical Care Medicine, Geisinger \\ Medical Center, Danville, USA 3. Cardiology, Guthrie Robert Packer Hospital, Sayre, USA 4. Critical Care Medicine, \\ Guthrie Robert Packer Hospital, Sayre, USA
}

Corresponding author: Mahin Rehman,drmahinrehman@gmail.com

\begin{abstract}
The COVID-19 pandemic is affecting millions across the globe. The population of immunosuppressed individuals are at greatest risk of morbidity and mortality. Data on COVID-19 induced illness in the immunocompromised host are sparse. We aim to highlight the possibility of atypical and non-respiratory presentations of COVID-19 (severe acute respiratory syndrome coronavirus 2, SARS-CoV-2) in immunosuppressed individuals as our case reveals a rare COVID-19 associated GI presentation of neutropenic enterocolitis with bloody diarrhea.
\end{abstract}

Categories: Gastroenterology, Infectious Disease, Oncology

Keywords: covid-19 pandemic, covid-19, sars-cov-2, neutropenic enterocolitis, chemotherapy, gastroenterology, enterocolitis, infectious disease, immunosuppression, immunocompromised

\section{Introduction}

The spectrum of the COVID-19 related disease manifestations is growing slowly. Primarily, the disease presents with a febrile illness and ultimately the development of respiratory symptoms with patients experiencing shortness of breath, tachypnea, and some decompensating into complete respiratory failure secondary to acute respiratory distress syndrome (ARDS) $[1,2]$. Aside from respiratory involvement, the disease has been linked to a thromboembolic state allowing individuals to have strokes and pulmonary emboli; it has also been linked to inducing myocardial injury whether in the form of myocarditis or myocardial infarction and has been linked to involvement of the gastrointestinal (GI) system [1,3-5].

Received 07/21/2020 Review began 07/22/2020 Review ended 07/23/2020 Published 07/28/2020

(๑) Copyright 2020 Rehman et al. This is an open access article distributed under the terms of the Creative Commons Attribution License CC-BY 4.0., which permits unrestricted use, distribution, and reproduction in any medium, provided the original author and source are credited.
COVID-19 is caused by the novel coronavirus severe acute respiratory syndrome coronavirus 2 (SARS-CoV2), and it is a single-stranded ribonucleic acid (RNA) virus with notable spike proteins [2]. The natural animal reservoir of this virus seems to be the chrysanthemum bat and this virus is highly virulent and easily transmissible via droplets, direct and indirect contact, and possibly airborne transmission [2]. The virus enters the cell through angiotensin-converting enzyme 2 receptors (ACE2), which is expressed in the lungs, heart, vessels, and GI tract [2-5]. The spike proteins facilitate entry into cells by binding to the ACE2 receptors [2]. The subsequent infection can lead to a cytokine release storm due to the imbalance of T-cell activation with dysregulated release of cytokines, such as interleukin (IL)-6, IL-17, etc., leading to a severe inflammatory response [2-4]. Recent data have revealed that severe cases of COVID-19 are associated with proinflammatory immune pathways and those patients who are in critical condition in the ICU due to COVID-19 had significantly higher concentrations of certain cytokines; additionally, the infection was noted to induce leucopenia and lymphopenia [6].

There have been reports of GI involvement, though significantly less compared to respiratory and cardiac compromise, with regards to mild symptoms. With regards to severe involvement, there are very limited cases such as hemorrhagic colitis and severe acute pancreatitis $[7,8]$. However, there are no reports of severe GI cases of COVID-19 in the immunosuppressed host.

\section{Case Presentation}

A 72-year-old male with a past medical history of poorly differentiated adenocarcinoma of the lower esophagus and gastroesophageal junction (stage III), type II diabetes, hypertension, hyperlipidemia, coronary artery disease, and atrial fibrillation on rivaroxaban is undergoing cancer treatment with a curative intent via neoadjuvant chemoradiation (carboplatin and taxol) weekly; his most recent chemotherapy treatment was one week prior to admission into the hospital, where he presented with nausea, vomiting, and diarrhea. He is found to be hypotensive (blood pressure $70 / 30 \mathrm{mmHg}$ ) and febrile (104 degrees Fahrenheit).

Lab work revealed him to be pancytopenic and his absolute neutrophil count (ANC) was low at $1.64 \mathrm{~K} / \mu \mathrm{L}$ (reference range 1.8-7.7 K/ $\mu \mathrm{L}$ ). Physical exam revealed clear breath sounds bilaterally and mild abdominal 


\section{Cureus}

tenderness in the right upper and lower quadrants with minimal distension. СТ angiography of the chest, abdomen, and pelvis revealed a significant amount of new portal venous gas with abnormal mucosal enhancement and edema related to the distal ileum, cecum, and ascending colon in conjunction with air seen within the mesenteric vessels, and no thrombosis was noted (Figure 1). This was highly concerning for bowel ischemia but an infectious etiology, such as neutropenic enterocolitis or typhlitis, was on the differential. Both the CT scan and chest x-ray endorsed clear lung fields with no acute cardiopulmonary pathologies described in COVID-19 infections. His bowel movements were frankly bloody, so prothrombin complex concentrate and fresh frozen plasma were administered to reverse the effects of rivaroxaban. His abdominal pain and distension worsened, and surgery was deemed necessary.

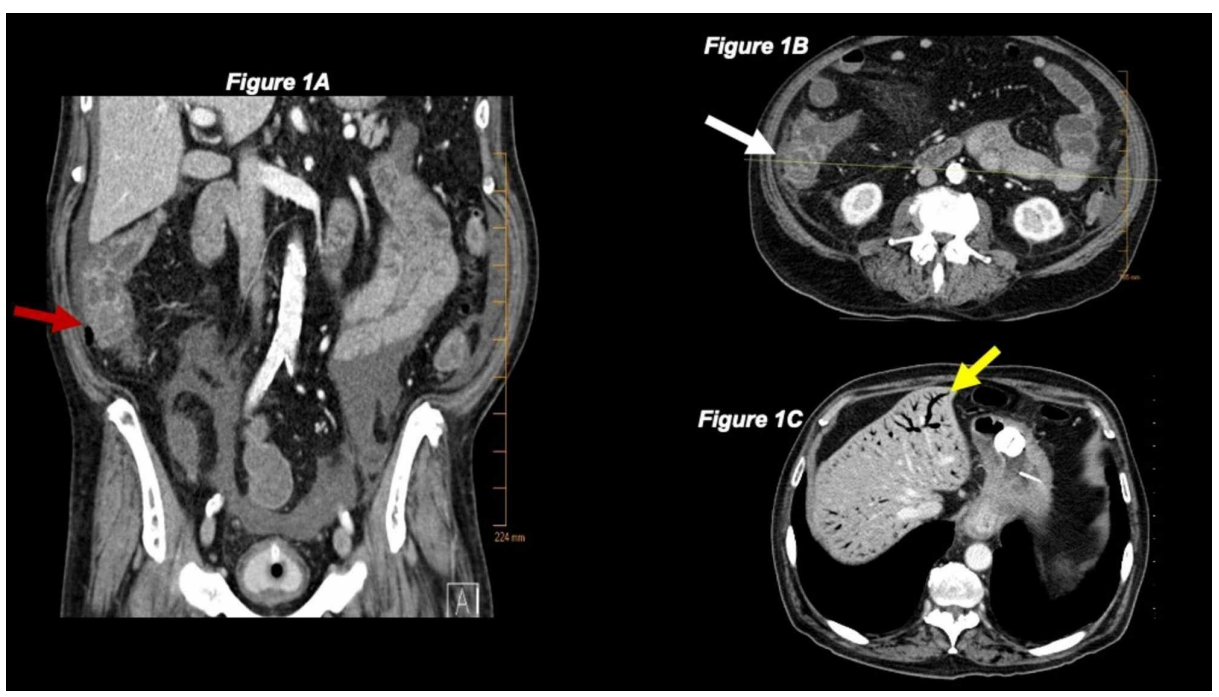

\section{FIGURE 1: CT of the abdomen revealing the site of neutropenic enterocolitis and portal venous gas}

(A) Coronal view of CT abdomen showing the site of neutropenic enterocolitis and gas in paracolic gutter (red arrow). (B) Transverse view of CT abdomen showing the site of neutropenic enterocolitis (white arrow). (C) Transverse view of CT abdomen showing significant portal venous gas that eventually spontaneously resolved within hours on repeat CT abdomen (yellow arrow).

Prior to surgery, SARS-CoV-2 RNA testing via nasopharyngeal swab was required, and he tested positive. Repeat CT scan prior to surgery was done to assess for mesenteric ischemia, and it showed spontaneous resolution of portal venous gas within hours of his initial CT; CT was negative for any mesenteric ischemia. His lab work was typical of an individual with an active COVID-19 infection: D-dimer $>20 \mathrm{mcg} / \mathrm{mL}$ (reference range $<0.5 \mathrm{mcg} / \mathrm{mL}$ ), C-reactive protein $21.7 \mathrm{mg} / \mathrm{dL}$ (reference range $<1 \mathrm{mg} / \mathrm{dL}$ ), ferritin $12,200 \mathrm{ng} / \mathrm{mL}$ (reference range 18-464 ng/mL), and lactate dehydrogenase 1,498 U/L (reference range 313-618 U/L) [6,9]. With an elevated procalcitonin of $5.69 \mathrm{ng} / \mathrm{mL}$ (reference range $<0.012 \mathrm{ng} / \mathrm{mL}$ ), a superimposed bacterial infection was suspected. The exploratory laparotomy showed a $90 \mathrm{~cm}$ long thickened ileus, consistent with neutropenic enterocolitis, and no evidence or signs of perforation, free air, bowel ischemia, obstruction, or purulent peritonitis were identified; the cause and source of gas noted on CT in the right paracolic gutter could not be identified. His bowels were deemed viable by the surgical team and resection was not performed. Blood cultures and stool studies including Clostridium difficile were negative; our institution did not have SARS-CoV-2 fecal testing. He failed to improve with IV broad-spectrum antibiotics, which is typically utilized in neutropenic patients with fever of unknown origin. Although neutropenic entercolitis allows bacteria to invade through the damaged gut wall because the host cannot mount an immune response against this, his failure to improve with antibiotics, despite being a non-specific sign, and worsening status made COVID-19 the most likely precipitating cause [10].

\section{Discussion}

COVID-19 manifests primarily as a respiratory disease although many infected individuals can be asymptomatic. Other manifestations have been reported that coincide with respiratory involvement, such as strokes, pulmonary embolisms, and myocardial injury associated with febrile illness and respiratory issues. GI symptoms occur in less than 10\% of patients who are infected with COVID-19 [11]. There have been very limited reports on bloody diarrhea and hemorrhagic colitis associated with COVID-19 [7]. This case of COVID-19 induced neutropenic enterocolitis with bloody diarrhea in an immunosuppressed host without respiratory involvement makes this a novel presentation.

Studies show that neutropenic enterocolitis usually develops with an ANC $<500 / \mu \mathrm{L}$ and it is extremely rare to develop this with an ANC $>1,000 / \mu \mathrm{L}$, which is another unique feature of COVID-19 induced enterocolitis 
$[12,13]$. To add to the rarity of this presentation, this individual presented with extensive portal venous gas concerning for bowel ischemia which managed spontaneously and rapidly (within a few hours) resolve on its own prior to being rule out via surgical exploration. He was managed conservatively postop and was successfully extubated one day after surgery to room air.

There are a couple of limitations to note in this case report. Firstly, our institution did not have the capability for fecal SARS-CoV-2 RNA testing to definitively confirm the presence of the virus in the gut. Secondly, chemotherapy itself can induce mucosal injury (mucositis) and lead possible distension and necrosis along with gut dysmotility and ileus; though it is extremely unlikely for chemotherapy itself to cause neutropenic enterocolitis directly without pathogenic involvement of microorganisms as bacterial translocation is common, it must still be considered [12]. Lastly, given that his bowels were deemed viable, no intraoperative biopsy or resection was performed so histological analysis of the bowel was not able to be conducted for definitive confirmation.

\section{Conclusions}

This case aims to bring light to physicians during this pandemic that not only are immunosuppressed individuals at greatest risk of morbidity and mortality, but that atypical and non-respiratory manifestations of COVID-19 in immunosuppressed individuals are indeed plausible. GI manifestations can occur in those infected with the SARS-CoV-2 virus as the GI tract does express the ACE2 receptor.

\section{Additional Information \\ Disclosures}

Human subjects: Consent was obtained by all participants in this study. Conflicts of interest: In compliance with the ICMJE uniform disclosure form, all authors declare the following: Payment/services info: All authors have declared that no financial support was received from any organization for the submitted work. Financial relationships: All authors have declared that they have no financial relationships at present or within the previous three years with any organizations that might have an interest in the submitted work. Other relationships: All authors have declared that there are no other relationships or activities that could appear to have influenced the submitted work.

\section{References}

1. Boukhris M, Hillani A, Moroni F, et al.: Cardiovascular implications of the COVID-19 pandemic: a global perspective. Can J Cardiol. 2020, 36:1068-1080. 10.1016/i.cjca.2020.05.018

2. ESC guidance for the diagnosis and management of CV disease during the COVID-19 pandemic . (2020). Accessed: July 20, 2020: https://www.escardio.org/Education/COVID-19-and-Cardiology/ESC-COVID-19Guidance.

3. Patel KP, Patel PA, Vunnam RR, Hewlett AT, Jain R, Jing R, Vunnam SR: Gastrointestinal, hepatobiliary, and pancreatic manifestations of COVID-19. J Clin Virol. 2020, 128:104386. 10.1016/j.jcv.2020.104386

4. Guo YR, Cao QD, Hong ZS, et al.: The origin, transmission and clinical therapies on coronavirus disease 2019 (COVID-19) outbreak - an update on the status. Mil Med Res. 2020, 7:11. 10.1186/s40779-020-00240-0

5. Wong SH, Lui RN, Sung JJ: Covid-19 and the digestive system. J Gastroenterol Hepatol. 2020, 35:744-748. 10.1111/jgh.15047

6. Huang C, Wang Y, Li X, et al.: Clinical features of patients infected with 2019 novel coronavirus in Wuhan, China. Lancet. 2020, 395:497-506. 10.1016/S0140-6736(20)30183-5

7. Carvalho A, Alqusairi R, Adams A, Paul M, Kothari N, Peters S, DeBenedet AT: SARS-CoV-2 gastrointestinal infection causing hemorrhagic colitis: implications for detection and transmission of COVID-19 disease. Am J Gastroenterol. 2020, 115:942-946. 10.14309/ajg.0000000000000667

8. Hadi A, Werge M, Kristiansen KT, Pedersen UG, Karstensen JG, Novovic S, Gluud LL: Coronavirus disease-19 (COVID-19) associated with severe acute pancreatitis:case report on three family members. Pancreatology. 2020, 20:665-667. 10.1016/j.pan.2020.04.021

9. Guan WJ, Ni ZY, Hu Y, et al.: Clinical characteristics of coronavirus disease 2019 in China . N Engl J Med. 2020, 382:1708-1720. 10.1056/NEJMoa2002032

10. Gorschlüter M, Mey U, Strehl J, et al.: Neutropenic enterocolitis in adults: systematic analysis of evidence quality. Eur J Haematol. 2005, 75:1-13. 10.1111/j.1600-0609.2005.00442.x

11. Sultan S, Altayar O, Siddique SM, et al.: AGA institute rapid review of the gastrointestinal and liver manifestations of COVID-19, meta-analysis of international data, and recommendations for the consultative management of patients with COVID-19. Gastroenterology. 2020, 159:320-334. 10.1053/j.gastro.2020.05.001

12. Rodrigues FG, Dasilva G, Wexner SD: Neutropenic enterocolitis. World J Gastroenterol. 2017, 23:42-47. 10.3748/wjg.v23.i1.42

13. Aksoy DY, Tanriover MD, Uzun O, et al.: Diarrhea in neutropenic patients: a prospective cohort study with emphasis on neutropenic enterocolitis. Ann Oncol. 2007, 18:183-189. 10.1093/annonc/mdl337 